Оригинальная статья/Original article

УДК 330

Методы оценки и управления предпринимательскими рисками

\begin{tabular}{lrl}
\hline \hline Ирина А. Киселева & 1 & kia1962@list.ru \\
Николай Е. Симонович & 2 & nsimoni@mail.ru \\
Галина Н. Егорова & 3 & egorovahp@gmail.com \\
Юрий А. Шаповалов & 3 & meatech@yandex.ru
\end{tabular}

$\overline{1}$ Российский экономический Университет имени Г.В. Плеханова, Стремянный пер., 36, г. Москва, Россия

${ }^{2}$ Российский Государственный Гуманитарный Университет, Миусская пл., 6, г. Москва, Россия

${ }^{3}$ Воронежский государственный университет инженерных технологий, пр-т Революции, 19, г. Воронеж, 394036, Россия

Реферат. Статья посвящена актуальной теме современности - управлению предпринимательскими рисками. Неотъемлемой составляющей профессионального управления рисками является определение сущности объекта управления в сфере экономики. Поскольку отечественная теория управления рисками находится в стадии формирования, то проблема четкого всестороннего определения понятия «риски» приобретает в настоящее время особую актуальность. В статье рассмотрены основные понятия риск-менеджмента; изучены его составляющие в предпринимательской деятельности; отражена система и принципы управления риском; исследованы основные виды рисков в сфере бизнеса. Предложен организационноэкономический механизм оценки предпринимательских рисков. Даны практические советы по управлению риском. Предпринимательства без рисков не существует. В условиях развития рыночной экономики конкретный предприниматель самостоятельно определяет методы, которыми будет осуществлять свою деятельность, и все они приводят к предпринимательским рискам. Уровень угроз на рынке сегодня выше уровня потенциальной прибыли. Сделан вывод о том, что невозможно повысить доход без увеличения риска или снизить риск без уменьшения дохода. Чем меньше диапазон вероятностного распределения ожидаемой доходности по отношению к ее средней величине, тем меньше риск, связанный с этой операцией. Избежать риска в предпринимательской деятельности практически невозможно, но можно уменьшить уровень этого риска. И это зависит от того, насколько профессионально и правильно действует сам предприниматель, какую стратегию он выберет, чтобы уменьшить появление рисков.

Ключевые слова: риск, оценка рисков, предпринимательские риски

\title{
Methods of assessment and management of enterprise risks
}

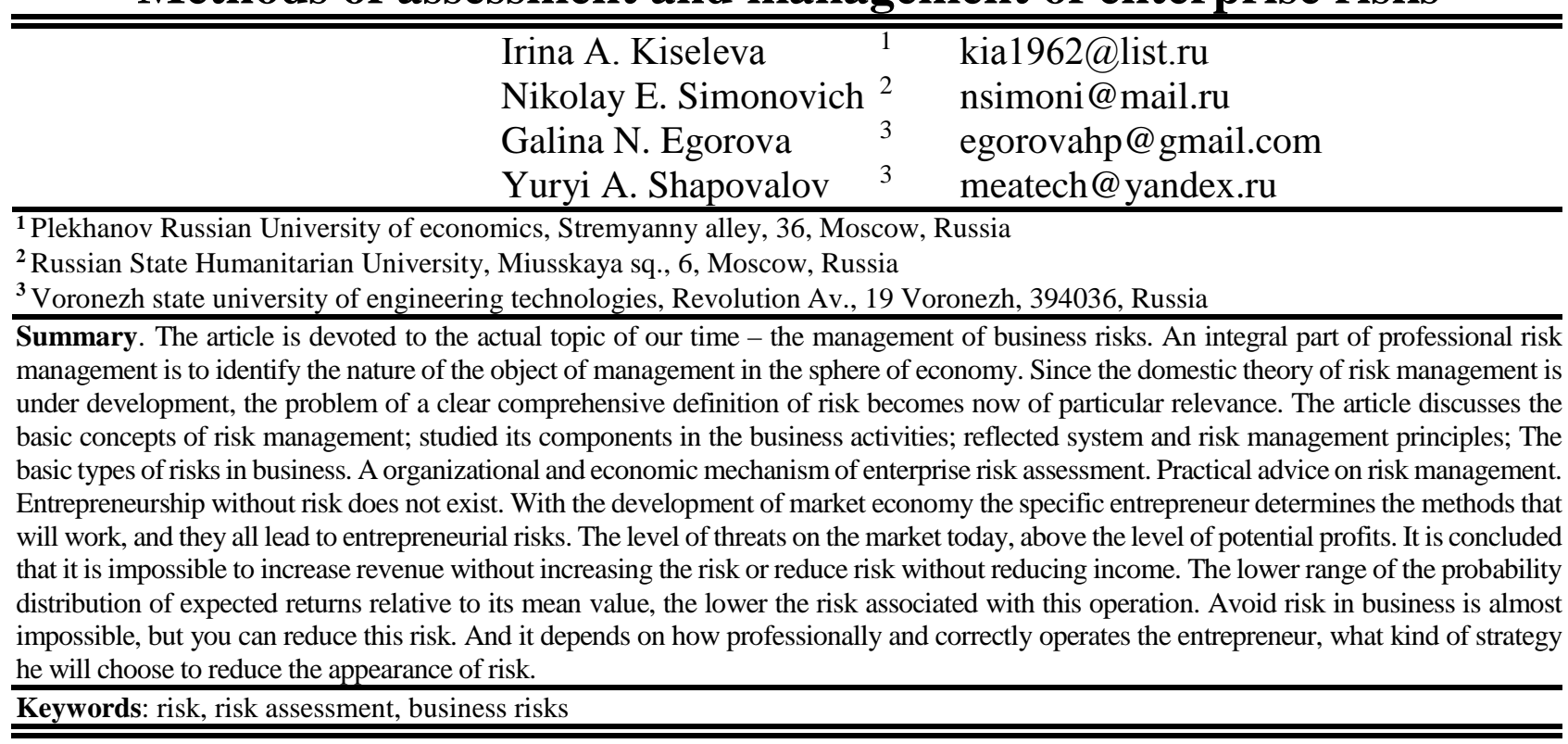

\section{Введение}

В странах с развитой экономикой существует множество наглядных примеров, когда предприятия, различные фирмы и организации терпят то подъем и процветание, то крах

\section{Для цитирования}

Киселева И.А., Симонович Н.Е., Егорова Г.Н., Шаповалов Ю.А. Методы оценки и управления предпринимательскими рисками // Вестник ВГУИТ. 2017. Т. 79. № 2. С. 314-319. doi:10.20914/23101202-2017-2-314-319 и банкротство вследствие своей деятельности. В среде жесткой конкуренции на рынке у каждого субъекта есть некий риск оказаться на дне своего жизненного цикла. И чтобы попытаться этого избежать, необходимо разобраться, какие методы снижения рисков существуют.

For citation

Kiseleva I.A., Simonovich N.E., Egorova G.N., Shapovalov Yu.A. Methods of assessment and management of enterprise risks. Vestnik VGUIT [Proceedings of VSUET]. 2017. vol. 79. no. 2. pp. 314-319. (in Russian). doi:10.20914/2310-1202-2017-2-314-319 


\section{Понятие и сущность предпринимательского риска}

Для того чтобы в полной мере понять и осмыслить тематику, заданную в настоящей работе, необходимо начать с самого понятия и сущности термина «предпринимательский риск». Итак, под предпринимательским риском, следует понимать невозможность предугадать и предусмотреть какое-либо событие и последствие этого события в дальнейшей деятельности предприятия. Риск - это всегда вероятность. Вероятность, что предприятие понесет убытки и неудачи ввиду своих решений [12, с. 85].

Многие предприниматели начинают открывать свой бизнес далеко не в легких и благоприятных условиях экономики страны. Кризис, который нарастает в настоящий момент в России, является началом того, что предприятия начинают усиливать предпринимательские риски. Вследствие этого увеличивается количество организаций, работающих себе в убыток [1, 3].

Обойти встречи с рисками в этой сфере деятельность невозможно. Умение рисковать это классическая черта, которая помогает предпринимателю противостоять неопределенности. Без нее невозможно построить успешно развивающийся бизнес. Чем больше риск, тем больше вероятность, что предприятие окажется на вершине своего жизненного цикла [8, с. 25].

В условиях рыночной среды, которая существует в наши дни, появляется все больше и больше причин, расширяющих территорию рисковых ситуаций. При таких обстоятельствах зарождается неуверенность в том, что конечный результат принесет положительный эффект предприятию. Поэтому и возрастает степень предпринимательского риска. Предпринимательские риски часто называют хозяйственными. Это означает, что они связаны с производственными, социальными, финансовыми и другими функциями.

\section{Виды предпринимательского риска}

К настоящему времени еще не разработана определенная классификация предпринимательских рисков. Из-за многообразия социальноэкономических отношений существует множество видов рисков в предпринимательской деятельности. Это связано с тем, что почти каждый день возникают какие-либо новые виды рисков. Более того, один и тот же вид можно просто определить различным термином [16, с. 128].

Но принято разграничивать виды предпринимательского риска с помощью факторов, вследствие которых они возникают. К примеру, политические факторы способствуют созданию политических рисков. Это может быть:
- Введение эмбарго (ограничение государством экспорта или импорта товаров, валюты);

- Национализация предприятия;

- Трудность осуществления предприятием своих действий из-за военных действий либо революций, нарастания негативного настроения в стране;

- Извлечение каких-либо товаров или предприятий с рынка.

Социально-экономические факторы являются причиной создания коммерческих (экономических) рисков. Они возникают в процессе реализации предпринимателем товаров и услуг. Здесь необходимо уметь учитывать многочисленные факторы (невыгодное увеличение цены закупаемых товаров, снижение цены реализации товаров и т. д.) $[11,15]$.

Такой фактор, как экологический, способствует возникновению еще одного вида рисков. Это производственный риск. Он связан с реализацией и созданием продукции (работы, услуги), а также с осуществлением иных видов производственной деятельности. Например, ограничение использования природных ресурсов приводит к тому, что начинает изменяться предпринимательская деятельность. Этот риск проявляется в остановке производства либо неритмичности работы предприятия, а также вследствие природных катастроф и аварий из-за наводнений, землетрясений и пожаров $[17,18]$.

Такие риски, как технические и инновационные, появляются вследствие научно-технических факторов. Они проявляются в следующем:

- В снижении дохода предприятия, ввиду разного рода факторов. Это могут быть: уменьшение фактических продаж по сравнению с планом, изменение \% ставки по кредиту и т. д.);

- В том, что предприниматели не сумели извлечь определенные экономические выгоды из своих действий;

- В экономических потерях, связанных, к примеру, с банкротством партнера.

\section{Методы оценки и управления предпринимательским риском}

В экономической науке до конца не разработаны методы оценки и управления предпринимательского риска, не существует четких инструкций по уменышению и предотвращению рисков. Хотя стоит отметить, что в последние годы появилось множество научных работ и статей, в которых при рассмотрении и анализа деятельности различных коммерческих организаций, затрагиваются вопросы о риске в предпринимательстве [2, 4-6, 13, 14]. 
Многообразие факторов хозяйственной деятельности предпринимателя - это основная причина того, что необходимо знать основные положения теории рисков, которые позволят предпринимателю найти нужный путь (методику) для оценки какого-либо риска [9, с. 67]. Риск можно объяснить с помощью таких критериев, как:

- Вероятность наступления события $P(E)$. Это отношение количества благоприятных исходов $(K)$ к общему числу всех исходов $(M)$,

$$
P(E)=K / M ; 0<P(E)<1 ;
$$

- Величина отклонения от прогнозируемого значения (размах вариации) $R$ - разница между $\max$ и min значением рассматриваемого фактора;

- Д-дисперсия;

- $M(E)$ - математическое ожидание;

- $K B$ - коэффициент вариации и др.

Управление рисками в деятельности предпринимателя связано с анализом всех факторов, связанных с внешней и внутренней средой хозяйственной деятельности $[19,20]$. В кругах российского предпринимательства крупные и средние предприятия слишком консервативны, поэтому им сложно реагировать на изменения во внутренней и внешней среде. В то время как малые предприятия способны быстро переориентироваться и уйти от убыточного бизнеса, предварительно проводя оценку степени риска (К приятия это является сложностью. Поэтому при управлении предприятием необходимо предусматривать последствия от принятия решения в сфере управления рисками [7, с. 85].

Существуют четыре зоны риска предпринимательской деятельности:

- I зона - не предвидится риск;

- II зона - риск допустим. Предпринимательская деятельность сохраняет свою экономическую целесообразность;

- III зона - критический риск (потери превышают прибыль и вложенные средства);

- IV зона - катастрофический риск (потери превышают критический уровень).

Чтобы избежать появления определенного вида риска, необходимо рассмотреть некоторые методы управления рисками. Их можно разделить на четыре категории. Все они представлены в таблице 1.

Таблица 1.

Types of risk management methods

Table 1.

Виды методов управления рисками

\begin{tabular}{|c|c|}
\hline $\begin{array}{c}\text { Название } \\
\text { метода } \mid \\
\text { Method name }\end{array}$ & $\begin{array}{c}\text { Сущность метода } \\
\text { The essence of the method }\end{array}$ \\
\hline $\begin{array}{lr}\text { Метод } & \\
\text { уклонения } & \text { от } \\
\text { риска | The } \\
\text { method of risk } \\
\text { evasion }\end{array}$ & $\begin{array}{l}\text { Необходимо просчитать все решения, связанные с управлением на стопроцентную гаран- } \\
\text { тию застрахованности от рисков. Этими решениями могут стать (It is necessary to calculate } \\
\text { all decisions related to management for a } 100 \% \text { risk insurance guarantee. These solutions can be): } \\
\text { - отказ от взаимодействия с партнерами, которые считаются ненадежными (refusal to coop- } \\
\text { erate with partners that are considered unreliable); } \\
\text { - отказ от инновационной деятельности (refusal of innovative activity); } \\
\text { - все виды страхования (all types of insurance); } \\
\text { - поиск надежных партнеров (search for reliable partners). }\end{array}$ \\
\hline $\begin{array}{l}\text { Метод } \\
\text { локализации } \\
\text { риска | Risk lo- } \\
\text { calization } \\
\text { method }\end{array}$ & $\begin{array}{l}\text { Необходимо выявить участников, которые в большей степени подвержены влиянию } \\
\text { факторов риска. В основе этого метода лежит выявление «опасных» зон в хозяйственной } \\
\text { деятельности предприятия, раскрутка бизнеса и т. д. | It is necessary to identify participants } \\
\text { who are more susceptible to the influence of risk factors. At the heart of this method lies the identification } \\
\text { of dangerous zones in the economic activities of enterprises, the promotion of business, and so on. }\end{array}$ \\
\hline $\begin{array}{l}\text { Метод } \\
\text { диссипации } \\
\text { риска | The } \\
\text { method of risk } \\
\text { dissipation }\end{array}$ & $\begin{array}{l}\text { Необходимо распределить факторы риска среди участников деятельности предприятия. } \\
\text { Принцип этого метода заключается в том, чтобы распеделить ответственность между } \\
\text { участниками. Это может быть образование АО, обмен акциями, расширение закупок } \\
\text { различных ресурсов, распределение риска по этапам работ. | It is necessary to distribute risk } \\
\text { factors among the participants of the enterprise activity. The principle of this method is to distrib- } \\
\text { ute responsibility among the participants. This can be the formation of joint stock companies, the } \\
\text { exchange of shares, the expansion of procurement of various resources, the distribution of risks } \\
\text { by the stages of work. }\end{array}$ \\
\hline $\begin{array}{l}\text { Название } \\
\text { метода | Method } \\
\text { name }\end{array}$ & $\begin{array}{l}\text { Это меры, инструменты, не способствующие зарождению рисков. Это методы стратегиче- } \\
\text { ского планирования и прогнозирования, борьба действиями в сфере криминала, создание } \\
\text { законопроектов, ликвидирующих появления факторов риска и т. д. I These are measures, } \\
\text { tools that do not promote the emergence of risks. These are methods of strategic planning and forecast- } \\
\text { ing, approving actions in the sphere of crime, creating bills, eliminating unforeseen risks, and so on. }\end{array}$ \\
\hline
\end{tabular}


В практике управление рисками подразделяют на следующие этапы:

- Подготовительный - распределение возможных альтернатив в условиях принятия решения, связанных с управлением предприятия;

- Организационный - предусматривает выбор некоторых методов управления рисками, способов, благодаря которым потери сойдутся к минимуму, ситуационного прогнозирования и планирования деятельности бизнеса (фирмы);

- Нормативный - создание инструкций для того, чтобы вывести предприятие из негативных ситуаций.

Следовательно, для того, чтобы организация находилась в комфортных условиях и ей не угрожала опасность в сфере защиты информации, потеря которой может повлечь за собой дальнейшие убытки, необходимо правильно профессионально организовать предпринимательскую деятельность [10, с. 142]. Стратегия экономической безопасности фирмы должна быть описана в соответствующих документах компании и быть частью стратегического развития фирмы.

В рамках этой стратегии должна сформироваться программа антирисковых мероприятий. Их могут проводить и сами работники предприятия, и приглашенные специалисты.

\section{Примеры ситуаций, которые приводят} к созданию факторов риска для фирмы

Приведем примеры, когда определенная ситуация приводит к созданию факторов риска для фирмы. И для каждой ситуации найдем оптимальный метод управления сложившегося риска. Примеры представлены в таблице 2.

Таблица 2.

Ситуации, приводящие к созданию факторов риска для фирмы и путь решения

Table 2 .

Situations leading to the creation of risk factors for the firm and the way to solve it

\begin{tabular}{|c|c|}
\hline \begin{tabular}{l|l} 
Ситуация | Situation \\
\end{tabular} & Решение | Decision \\
\hline $\begin{array}{l}\text { Напряженная политическая ситуация в стране, вслед- } \\
\text { ствие этого развитие частного предпринимательства } \\
\text { находится на очень низком уровне | The tense political } \\
\text { situation in the country, as a result, the development of pri- } \\
\text { vate entrepreneurship is at a very low level }\end{array}$ & $\begin{array}{l}\text { Необходим метод компенсации рисков (стратегическое } \\
\text { планирование, прогнозирование). Этот метод описан в } \\
\text { таблице } 1 \text {. | A method of risk compensation is needed (strategic } \\
\text { planning, forecasting). This method is described in table } 1 \text {. }\end{array}$ \\
\hline $\begin{array}{l}\text { Низкий жизненный уровень населения страны приводит к } \\
\text { падению платежеспособного спроса | Low living standards of } \\
\text { the population lead to a fall in effective demand }\end{array}$ & $\begin{array}{l}\text { Метод уклонения от рисков (страхование, отказ от } \\
\text { инноваций)| The method of avoiding risks (insurance, rejec- } \\
\text { tion of innovations) }\end{array}$ \\
\hline $\begin{array}{l}\text { На рынке появились новые конкуренты | New } \\
\text { tors appeared on the market }\end{array}$ & $\begin{array}{l}\text { Метод диссипации. Следует произвести увеличение } \\
\text { числа наименований и видов изделий, выявить «опасные» } \\
\text { с точки зрения экономики сектора рынка. | The dissipation } \\
\text { method. It is necessary to increase the number of items and } \\
\text { types of products, identify dangerous from the point of view } \\
\text { of the economy of the market sector. }\end{array}$ \\
\hline
\end{tabular}

\section{Заключение}

Любое предприятие рано или поздно может понести определенные риски, которые связаны с производственной и коммерческой деятельностью предприятия. Избежать риска в предпринимательской деятельности практически невозможно, но можно уменьшить уровень этого риска. И это зависит от того, насколько профессионально и правильно действует сам предприниматель, какую стратегию он выберет, чтобы уменьшить появление рисков. Организация

\section{ЛИТЕРАТУРА}

1 Альгин А.П. Грани экономического риска. М.: Знание, 1991.

2 Акаев А.А., и др. Проекты и риски будущего: концепции, модели, инструменты, прогнозы, M.: URSS, 2011. $432 \mathrm{c}$.

3 Гитман Л.Дж., Джонк М.Д. Основы инвестирования, М. Дело, 1997. с.

4 Власов А. Риск-менеджмент: система управления потенциальными потерями // Бизнес. 2013. № 5. C. 2013. развития деятельности предприятия основывается на профессиональном знании, умении и навыков работы в различных условиях экономической ситуации. Поэтому знания в сфере управления рисками необходимы для организации успешно развивающегося бизнеса. Между динамикой уровня риска и динамикой доходности существует прямая зависимость, а именно: чем выше средняя доходность операции, тем выше связанный с нею риск. Практически невозможно повысить доход без увеличения риска или снизить риск без уменьшения дохода.

5 Дорохина Е.Ю. Риски проектов: теория и практика управления // Вестник Российского экономического университета им. Г.В. Плеханова. 2009. № 6. C. 86-93.

6 Дорохина Е.Ю. Ресурсы покрытия рисков на предприятии // РИСК: Ресурсы информация снабжение конкуренция. 2009. № 3. С. 116-120.

7 Киселева И.А., Симонович Н.Е. Особенности оценки рисков и роль мотивации в период кризиса, М: Новая реальность, 2016. 136 с. 
8 Киселева И.А., Симонович Н.Е. Принятие решений в условиях риска: психологические аспекты // Финансовая аналитика: проблемы и решения. 2014. № 18. С. 23-29.

9 Киселева И.А., Симонович Н.Е. Проблемы безопасности и риска с позиции психолога и экономиста, М: Новая реальность, 2016. 148 с.

10 Леонтьева Л.С., Кузнецов В.И. Производственный менеджмент М.: Юрайт, 2014. 305 с.

11 Норткотт Д. Принятие инвестиционных решений, М.: Банки и биржи ЮНИТИ, 1997.

12 Тихомиров Н.П., Тихомирова Т.М. Риск анализ в экономике, М.: Экономика, 2010. 168 с.

13 Тутова Н.А. Каждый из видов риска необходимо держать под жестким контролем // Банковские технологии, 2013. № 2. с. 30.

14 Халиков М.А., Хечумова Э.А., Шардин А.А. Методология учета и оценки рисков производственной и финансовой сфер деятельности предприятия // Ученые записки Российской Академии предпринимательства. 2010. № 23. С. 165-180.

15 Черешкин Д.С. Управление рисками и безопасностью М.: Издательская группа URSS, 2010. $200 \mathrm{c}$.

16 Экономическая теория. Концептуальные основы и практика / Под общ. ред. В.Ф. Максимовой М.: ЮНИТИДАНА, 2012. 751 с.

17 Financial optimization. Edited by Stavros A. Zeios. Cambridge University Press, 1993.

18 Alexander C. Financial Risk Management and Analysis. Wiley, 1996.

19Financial Risk Management and Analysis. Wiley, 1996.

20 Vaugham E.J. Risk management. N.Y. etc.: Wiley, 1997.

21 Мистров Л.Е., Дерканосова А.А. Метод информационного воздействия при синтезе стратегии управления конкурентоустойчивостью социально-экономических организаций // Вестник ВГУИТ. 2013. 2013. № 4 (58). С. 282-288.

22 Matveev V.V. Monitoring of economic risks of business activities // Russian Journal of Agricultural and Socio-Economic Sciences. 2016. T. 55. № 7. C. 72-79.

\section{REFERENCES}

1 Al'gin A.P. Grani ekonomicheskogo riska. [The verge of economic risk.]. Moscow, Znanie 1991. p. (in Russian).

2 Akaev A.A., i dr. Proekty i riski budushchego: kontseptsii, modeli, instrumenty, prognozy [Projects and risks of the future: concepts, models, instruments, forecasts]. Moscow, URSS, 2011. 432 p. (in Russian).

3 Gitman L.Dzh., Dzhonk M.D. Osnovy investirovaniya [Investing basics]. Moscow, Delo, 1997. (in Russian).

4 Vlasov A. Risk management: control system potential losses. Biznes [Business]. 2013. no. 5. 2013. (in Russian).

5 Dorokhina E.Yu. Project risks: the theory and practice of management. Vestnik Rossiiskogo ekonomicheskogo universiteta [Proceedings of the Russian economic University]. 2009. no. 6. pp. 86-93. (in Russian).
6 Dorokhina E.Yu. Resources cover risks in the enterprise. RISK: Resursy informatsiya snabzhenie konkurentsiya [RISK: Resources, information, supply, competition]. 2009. no. 3. pp. 116-120. (in Russian).

7 Kiseleva I.A., Simonovich N.E. Osobennosti otsenki riskov i rol' motivatsii v period krizisa [Features of the risk assessment and the role of motivation in times of crisis]. Moscow, Novaya real'nost', 2016. 136 p. (in Russian).

8 Kiseleva I.A., Simonovich N.E. Decision making under risk: psychological aspects. Finansovaya analitika: problemy i resheniya [Financial Analytics: problems and solutions]. 2014. no. 18. pp. 23-29. (in Russian).

9 Kiseleva I.A., Simonovich N.E. Problemy bezopasnosti i riska s pozitsii psikhologa i ekonomista [Security concerns and risk from the perspective of a psychologist and economist]. Moscow, Novaya real'nost', 2016. 148 p. (in Russian).

10 Leont'eva L.S., Kuznetsov V.I. Proizvodstvennyi menedzhment [Production management]. Moscow, Yurait, 2014. 305 p. (in Russian).

11 Nortkott D. Prinyatie investitsionnykh reshenii [Investment decisions]. Moscow, Banki i birzhi YuNITI, 1997. (in Russian).

12 Tikhomirov N.P., Tikhomirova T.M. Risk analiz $\mathrm{v}$ ekonomike [Risk analysis in Economics]. Moscow, Ekonomika, 2010. 168 p. (in Russian).

13 Tutova N.A. Each of the types of risk must be kept under tight control. Bankovskie tekhnologii [Bank technologies]. 2013. no. 2. pp. 30. (in Russian). 14 Khalikov M.A., Khechumova E.A., Shardin A.A. Accounting and risk assessment operational and financial areas of the company. Uchenye zapiski Rossiiskoi Akademii predprinimatel'stva [Scientific notes of the Russian Academy of entrepreneurship]. 2010. no. 23. pp. 165-180. (in Russian).

15 Chereshkin D.S., bezopasnost'yu Upravlenie riskami i bezopasnost'yu [Risk management and safety]. Moscow, Izdatel'skaya gruppa URSS, 2010. 200 p. (in Russian).

16Ekonomicheskaya teoriya. Kontseptual'nye osnovy i praktika [Economic theory. The conceptual basis and practice. Edited by V. F. Maximova]. Moscow, YuNITIDANA, 2012. 751 p. (in Russian).

17 Financial optimization. Edited by Stavros A. Zeios. Cambridge University Press, 1993.

18 Alexander C. Financial Risk Management and Analysis. Wiley, 1996.

19Financial Risk Management and Analysis. Wiley, 1996.

20 Vaugham E.J. Risk management. N.Y. etc .: Wiley, 1997.

21 Mistrov L.E., Derkanosova A.A. The method of the information impact in the synthesis of the control strategy the stability of socio-economic organizations.. Vestnik VGUIT [Proceedings of VSUET]. 2013. no. 4 (58). pp. 282-288. (in Russian).

22 Matveev V.V. Monitoring of economic risks of business activities. Russian Journal of Agricultural and Socio-Economic Sciences. 2016. vol. 55. no. 7. pp. 72-79. (in Russian). 


\section{СВЕДЕНИЯ ОБ АВТОРАХ}

Ирина А. Киселева д.э.н., профессор, кафедра математических методов в экономике, Российский экономический Университет имени Г.В. Плеханова, Стремянный пер., 36, г. Москва, Россия, kia1962@list.ru

Николай Е. Симонович д.псх.н., профессор, кафедра психологии личности Института психологии им. Л.С. Выготского, Российский Государственный Гуманитарный Университет, Миусская пл., 6, г. Москва, Россия, nsimoni@mail.ru

Галина Н. Егорова к. п. н., доцент, кафедра машин и аппаратов химических производств, Воронежский государственный университет инженерных технологий, пр-т Революции, 19, г. Воронеж, 394036, Россия, egorovahp@gmail.com

Юрий А. Шаповалов студент, кафедра технологии продуктов животного происхождения, Воронежский государственный университет инженерных технологий, пр-т Революции, 19, г. Воронеж, 394036, Россия, meatech@yandex.ru

\section{КРИТЕРИЙ АВТОРСТВА}

Все авторы в равной степени принимали участие в написании рукописи и несут ответственность за плагиат

КОНФЛИКТ ИНТЕРЕСОВ

Авторы заявляют об отсутствии конфликта интересов.

ПОСТУПИЛА 01.04.2017

ПРИНЯТА В ПЕЧАТЬ 01.05.2017

\section{INFORMATION ABOUT AUTHORS}

Irina A. Kiseleva doctor of economical sciences, professor, mathematical methods in economics department, Plekhanov Russian University of economics, Plekhanov Russian University of economics, Stremyanny alley, 36, Moscow, Russia,kia1962@list.ru

Nikolay E. Simonovich doctor of psychological sciences, professor, department of personality psychology institute named after L.S. Vygotsky, Russian State Humanitarian University, Miusskaya sq., 6, Moscow, Russia, nsimoni@mail.ru

Galina N. Egorova candidate of pedagogical sciences, assistant professor, machines and apparatuses of chemical production department, Voronezh state university of engineering technologies, Revolution Av., 19 Voronezh, 394036, Russia, egorovahp@gmail.com

Yuryi A. Shapovalov student, technology of animal products, Voronezh state university of engineering technologies, Revolution Av., 19 Voronezh, 394036, Russia, meatech@yandex.ru

\section{CONTRIBUTION}

All authors equally took part in writing the manuscript and are responsible for plagiarism

\section{CONFLICT OF INTEREST}

The authors declare no conflict of interest.

RECEIVED 4.1.2017

ACCEPTED 5.1.2017 